\title{
Probing complexes with single fluorophores: factors contributing to dispersion of FRET in DNA/RNA duplexes
}

\author{
Dmitry I. Cherny · Ian C. Eperon • \\ Clive R. Bagshaw
}

Received: 24 July 2008/Revised: 23 October 2008/Accepted: 23 October 2008/Published online: 18 November 2008

(C) The Author(s) 2008. This article is published with open access at Springerlink.com

\begin{abstract}
Single molecule fluorescent microscopy is a method for the analysis of the dynamics of biological macromolecules by detecting the fluorescence signal produced by fluorophores associated with the macromolecule. Two fluorophores located in a close proximity may result in Förster resonance energy transfer (FRET), which can be detected at the single molecule level and the efficiency of energy transfer calculated. In most cases, the experimentally observed distribution of FRET efficiency exhibits a significant width corresponding to $0.07-0.2$ (on a scale of $0-1$ ). Here, we present a general approach describing the analysis of experimental data for a DNA/RNA duplex. We have found that for a $15 \mathrm{bp}$ duplex with $\mathrm{Cy} 3$ and $\mathrm{Cy} 5$ fluorophores attached to the opposite ends of the helix, the width of the energy transfer distribution is mainly determined by the photon shot noise and the orientation factor, whereas the variation of inter-dye distances plays a minor role.
\end{abstract}

Keywords Fluorescence · FRET - Single molecule · Flexibility $\cdot$ DNA/RNA duplex

Electronic supplementary material The online version of this article (doi:10.1007/s00249-008-0383-z) contains supplementary material, which is available to authorized users.

D. I. Cherny is on leave from the Institute of Molecular Genetics, Russian Academy of Sciences, Moscow, Russia

D. I. Cherny $(\varangle) \cdot$ I. C. Eperon · C. R. Bagshaw

Department of Biochemistry, University of Leicester,

Leicester LE1 9HN, UK

e-mail: dc114@le.ac.uk

\section{Introduction}

The dynamics of biological macromolecules, including DNA, RNA and proteins, play a role in all cellular processes. A sensitive tool for monitoring the assembly of macromolecules and their kinetics is fluorescence microscopy, by virtue of imaging the dynamics of fluorescent dyes attached to the molecules of interest. Recently, fluorescent imaging techniques have been developed for individual molecules, allowing the monitoring of molecular interactions at the single molecule level (Blanchard et al. 2004; Coban et al. 2006; Deniz et al. 1999; Dietrich et al. 2002; Friedman et al. 2006; Gell et al. 2006; Ha 2001, 2004; Merchant et al. 2007; Murphy et al. 2004; Myong et al. 2006; Rueda et al. 2004; Yasuda et al. 2003). These new techniques provide unique information about the natural heterogeneity (structural and dynamics) of individual macromolecules, and allow the analysis of interactions between the molecules, the dynamics of complex formation, determination of the stoichiometry of the complex and calculation of the spatial distance between specific molecules in a complex.

The spatial distance between two molecules within a single complex, or more exactly the distance between two fluorophores, can be calculated by determining the positions for each fluorophore upon specific excitation of each. Under appropriate conditions, the fluorophores can be located with an accuracy of $1 \mathrm{~nm}$ (Churchman et al. 2005; Thompson et al. 2002; Yildiz and Selvin 2005), thus allowing reliable measurements of inter-dye distances usually in the range of $10-40 \mathrm{~nm}$ (i.e., a co-localization technique). When two dyes come into a closer proximity (less than $10 \mathrm{~nm}$ ) and the donor emission spectra overlaps with the acceptor absorption spectra, a fraction of donorexcited energy may be transferred to an acceptor via 
dipole-dipole interactions (Förster resonance energy transfer, FRET) leading to fluorescence of the acceptor (FRET imaging) without direct excitation of the latter. Since the rate of energy transfer has a very strong dependence on the distance between the dyes, i.e.,

$E=\frac{1}{1+\left(\frac{R}{R_{0}}\right)^{6}}$,

FRET is a very sensitive tool for determining distances, usually in the range of 2-8 $\mathrm{nm}$.

Single molecule FRET has been applied to a number of macromolecules, e.g., DNA, RNA, proteins and their complexes, providing unique information about the structure and dynamics of macromolecules and of the complexes that is unattainable from bulk measurements. In many cases, the information about inter-dye distance is extracted from FRET efficiency histograms which are often approximated by a Gaussian function (Agrawal et al. 2008; Coban et al. 2006; Deniz et al. 1999; Dietrich et al. 2002; Ha 2001; Iqbal et al. 2008a; Kapanidis et al. 2004; Koopmans et al. 2007; Kuzmenkina et al. 2006; Lee et al. 2005; Merchant et al. 2007; Murphy et al. 2004; Nir et al. 2006; Sabanayagam et al. 2005; Schuler et al. 2005; Sugawa et al. 2007; Yildiz and Selvin 2005; Yim et al. 2005; Zhang et al. 2007). The apparent width of thus extracted histograms is usually in the range of $0.07-0.2$ (on a scale of 0-1) and often ascribed to variations of the interdye distance (Coban et al. 2006; Dietrich et al. 2002; Merchant et al. 2007; Schuler et al. 2005; Sugawa et al. 2007). Here we describe an approach to discern the contributions of various factors to the apparent width of FRET distribution, showing that photon shot noise and modulations of orientation factor due to helix flexibilities are the main sources for the experimentally observed width of the FRET distribution.

\section{Materials and methods}

\section{Preparation of DNA/RNA duplex}

Oligonucleotides Cy3-5'-ACCUGCAGGCAUGCA-3' (2'OMe bases) and Cy5-5'-TGCATGCCTGCAGGT-3'biotin (DNA bases) were obtained from Eurogentec, Belgium. Briefly, oligonucleotides were synthesized using phosphoramidite chemistry. Cy3, Cy5 and biotin (Glen Research reagents) were coupled via hexanediol spacer C6. The samples were PAGE purified, electroeluted, desalted and lyophilized. Lyophilized samples were reconstituted in $10 \mathrm{mM}$ Tris-HCl, $0.1 \mathrm{mM} \mathrm{Na} \mathrm{NaDTA}_{3}$, pH 8.0. To prepare DNA/RNA duplex a mixture containing both oligonucleotides $(\sim 1 \mu \mathrm{M}$ each $)$ in a buffer A (10 mM Hepes, $\mathrm{pH} 8.0$,
$100 \mathrm{mM} \mathrm{NaCl}$ ) was heated at $80^{\circ} \mathrm{C}$ for $5-10 \mathrm{~min}$, then slowly cooled down to $50^{\circ} \mathrm{C}(\sim 1.5 \mathrm{~h})$, and finally placed on ice for 1.5-2 $\mathrm{h}$. The absence of non-annealed oligonucleotides was checked by agarose gel (2\%, TAE buffer) electrophoresis using a PhosphorImager 9400 (Typhoon, GE Healthcare) as the imaging device.

Preparation of the sample for single molecule detection

To detect the fluorescence signal from the sample we used a flow chamber built between a glass cover slip $(0.1 \mathrm{~mm})$ and quartz slide $(1 \mathrm{~mm})$ separated by a spacer $(0.1 \mathrm{~mm})$ as described (Conibear and Bagshaw 2000). The quartz surface was modified by PEG/PEG-biotin treatment as described (Ha et al. 2002). Then $25 \mu \mathrm{l}$, of streptavidin solution at $0.1 \mathrm{mg} / \mathrm{ml}$ in PBS $\left(8 \mathrm{mM} \mathrm{Na} \mathrm{HPO}_{4}, 1 \mathrm{mM}\right.$ $\left.\mathrm{KH}_{2} \mathrm{PO}_{4}, 2.7 \mathrm{mM} \mathrm{KCl}, 137 \mathrm{mM} \mathrm{NaCl}, \mathrm{pH} 7.4\right)$ was injected into the fluid chamber for 5-10 min, after when the chamber was flushed with PBS buffer. $25-30 \mu$ of the DNA/RNA duplex was diluted into buffer $A$ to a final concentration $5-20 \mathrm{pM}$ and injected into the chamber. After 5-10 min, when a sufficient number of fluorescent spots could be imaged on both halves of the camera chip, the chamber was flushed with $25-50 \mu \mathrm{l}$ with PBS and finally was rinsed with scavenger buffer (Ha et al. 2002).

Detection of fluorescence signals

Single molecule experiments were performed using a custom-built prism-based total internal fluorescence microscope (Conibear and Bagshaw 2000), using a $532 \mathrm{~nm}$ laser (Suwtech $50 \mathrm{~mW}$ DPSS, sp3plus, Tunbridge Wells, Kent UK) for excitation with an incident power (at the prism) of $\sim 100 \mathrm{~W} / \mathrm{cm}^{2}$ (corresponding to $\sim 3 \times 10^{16}$ photons/s or $\sim 2.5 \times 10^{4}$ photons $/ \AA^{2} \mathrm{~s}$, illumination area is $\sim 10^{4} \mu \mathrm{m}^{2}$ ) throughout the experiments. Fluorescence emission was collected by a $63 \times 1.2 \mathrm{NA}$ Zeiss C-apochromat water immersion lens, split by a dichroic mirror (645 DRLP, Omega) and projected onto two halves of the detector chip (iXon DV887 emCCD camera, Andor Technology, UK) through emission filters specific for Cy3 and Cy5 chromophores (580 DF30 and 670 DF40, Omega, respectively) using a home-built beam splitter. The acquisition rate was 100 or $900 \mathrm{~ms}$ per frame. The camera conversion factor (counts/photon) was determined by fitting of the probability density function of the camera count numbers as described (Ulbrich and Isacoff 2007) and found to be $\sim 7.5$. The spatial resolution of the microscope was $\sim 130 \mathrm{~nm} /$ pixel in the imaging plane.

Ensemble fluorescence spectra and steady-state anisotropies were measured on an SLM 8000 spectrofluorimeter (Amino, Urbana, IL, USA). 


\section{Data processing}

For each time series, an accumulated image was calculated to identify co-localized spots exhibiting FRET from the comparison of both halves of the accumulated image. Since the apparent width of the spots (FWHM of the fitted Gaussian) is $\sim 3$ pixels, an area $10 \times 10$ pixels was selected for the calculation of time series intensities. The raw signals for donor, $F_{\mathrm{D}}$, and acceptor, $F_{\mathrm{A}}$, were corrected for the corresponding background resulting in $I_{D}$ and $I_{A}$ signals, respectively. The mean values for background $I_{\mathrm{bg}}, \mathrm{D}$ and $I_{\mathrm{bg}}, \mathrm{A}$, were calculated from the corresponding Gaussian fits. The mean values for donor and acceptor intensities were also calculated from the corresponding Gaussian fits (where indicated). We used two approaches for the calculation of FRET efficiency. First, FRET was calculated from the reduction in donor fluorescence during energy transfer as follows

$E_{1}=1-\frac{I_{\mathrm{D}}}{\left\langle I_{\mathrm{D}, \text { noFRET }}\right\rangle}$

where $I_{\mathrm{D}}$ is the donor intensity during FRET and $<I_{\mathrm{D} \text {,noFRET }}>$ is the mean intensity of the donor after acceptor bleaching. The FRET histogram extracted from time traces was approximated by a Gaussian to yield the mean values for FRET and its dispersion. Since $I_{\mathrm{D}}$ signal is weak when FRET occurs, thus calculated FRET histogram is usually broader in comparison to the one calculated using another approach (see below), although the mean values are very similar.

Another approach for the calculation of FRET efficiency is based on simultaneous measuring of fluorescence of acceptor (due to FRET) and donor. This needs measurements of the leakage of donor signal into acceptor channel, $\omega$, and a correction factor, $\gamma$, accounting for the difference in quantum yields of donor and acceptor, together with detection efficiencies of the microscope for donor and acceptor signals (Kapanidis and Weiss 2002; Lee et al. 2005). The leakage factor was calculated as

$\omega=\frac{I_{\mathrm{A}, \text { noFRET }}}{\left\langle I_{\mathrm{D}}^{0}\right\rangle}$.

The corresponding histogram has a Gaussian-like shape thus allowing estimation of the mean of $\omega,\langle\omega\rangle$, from the Gaussian fit. For the given microscope setup and the Cy3Cy5 pair, $<\omega>$ was 0.18 . The leakage factor was consistent for all the molecules and served as an additional control for choosing the time interval corresponding to the total acceptor bleaching. The $\gamma$ factor is defined as

$\gamma=\frac{I_{\mathrm{A}}-\langle\omega\rangle I_{\mathrm{D}}}{\left\langle I_{\mathrm{D}, \text { noFRET }}\right\rangle-I_{\mathrm{D}}}$

The cumulated histograms for $\gamma$ showed a broad distribution which is significantly skewed for small rates of photon flux; however, it became Gaussian-like for larger acquisition rates. Experimentally, $\gamma$ is determined as

$\langle\gamma\rangle=\frac{\left\langle I_{\mathrm{A}}\right\rangle-\langle\omega\rangle\left\langle I_{\mathrm{D}}\right\rangle}{\left\langle I_{\mathrm{D}, \text { noFRET }}\right\rangle-\left\langle I_{\mathrm{D}}\right\rangle}$

for each molecule. Having calculated $\langle\omega\rangle$ and $\langle\gamma\rangle$, the FRET efficiency distribution was calculated as

$E_{2}=\frac{I_{\mathrm{A}}-\langle\omega\rangle I_{\mathrm{D}}}{I_{\mathrm{A}}-\langle\omega\rangle I_{\mathrm{D}}+\langle\gamma\rangle I_{\mathrm{D}}}$

Since the FRET distribution can be approximated by a Gaussian (Gopich and Szabo 2005), the mean FRET efficiency and its dispersion were extracted from the corresponding Gaussian fit and used for the analysis.

We note that the goodness of the Gaussian fit used for calculating the mean values $\left(I_{\mathrm{bg}, \mathrm{A}}, I_{\mathrm{bg}, \mathrm{D}}, I_{\mathrm{A}}, I_{\mathrm{D}}, \gamma, \omega\right)$ was checked by a reduced $\chi^{2}$ and in most cases was within $5 \%$ of the significance level.

Simulation of photon shot noise

Shot noise due to photon statistics was simulated using procedures described (Antonik et al. 2006; Gopich and Szabo 2005; Nir et al. 2006). Briefly, we selected the number of photons corresponding to the sum of those detected in both channels for either acquisition rate, i.e., 25 or 225 photons on average. The number of photons that can be detected, for instance, in a donor channel was calculated using a binomial distribution and the apparent FRET efficiency, $e$, as described (Antonik et al. 2006, formulae 3, 4 and 8). Therefore, the number of photons detected in an acceptor channel is equal to the difference between the total number of photons and those detected in a donor channel. The number of photoelectrons and the corresponding noise were calculated using a gain factor together with an additional noise factor. Camera off-set noise (for each half of the imaging chip) was generated and added to the simulated signals. The conventional FRET efficiency, $E$, that is experimentally measured, was calculated as proposed (Antonik et al. 2006)

$e=1-\frac{1}{1+\gamma \frac{E}{1-E}+\omega}$

where $\gamma$ and $\omega$ stand for the correction and the leakage factors, respectively (see above). The variance of the total number of photons (around the mean) together with the variance of the photons detected in either channel and camera noise lead to dispersion in the FRET histogram. Finally, the FRET histogram so obtained was fitted with a Gaussian providing the mean of FRET and its dispersion. Adjusting the parameters $e$ and $\gamma$ ( $\omega$ was set to 0.18 ) allowed matching of the average number photons detected 
in both channels and the mean value of FRET obtained experimentally and from simulation experiments.

Photon shot noise (detected as photoelectrons) in a strict sense is not constant across the $10 \times 10$ pixel area used for the experimental calculation of spot intensity, but it differs for each pixel, $(i, j)$, where $i, j=1,2, \ldots, 10$. The variance of noise for any pixel, $\sigma_{i, j}$, is equal to

$\sigma_{i, j}^{2}=F^{2} G^{2}\left(D_{\mathrm{QE}} n_{i, j}+\sigma_{\text {dark }}^{2}+\sigma_{\text {cic }}^{2}\right)+\sigma_{\text {readout }}^{2}$

(http://www.andor.com) where $G$ is the gain factor of the camera, $F$ is the noise factor, $D_{\mathrm{QE}}$ is the quantum efficiency of the detector, $n_{i, j}$ is the number of incident photons that hit $(i, j)$ pixel, $\sigma_{\text {dark }}$ accounts for the dark current noise, $\sigma_{\text {cic }}$ accounts for the clock induced charge noise and $\sigma_{\text {readout }}$ accounts for the readout noise. However, as we count the total number of photons, $N$, per analyzed area, i.e., $N=$ $\sum_{i, j=1}^{10} n_{i, j}$, the variance of the total noise will be the sum of variances for each pixel. It means that $\sigma_{\text {tot }}^{2}=F^{2} G^{2} D_{\mathrm{QE}} N+$ $F^{2} G^{2} S\left(\sigma_{\text {dark }}^{2}+\sigma_{\text {cic }}^{2}\right)+S \sigma_{\text {readout }}^{2}$, where $S$ is the size of the analyzed area. Since $D_{\mathrm{QE}}$ is close to unity, the above formula can be reduced to $\sigma_{\text {tot }}^{2} \approx F^{2} G^{2} N+S A$, where $A$ is a constant. The parameters $G$ and $A$ are determined experimentally, and the parameter $F$ is equal to two as described by the manufacturer. Together, it means that for the simulation of the total noise, i.e, fluctuations of photoelectrons, we need to know the total number of incident photons per spot (or analyzed area) only.

\section{Results}

Typical time trajectories for the fluorescence signals produced by donor (Cy3) and acceptor (Cy5) attached to the ends of DNA/RNA duplex (see Fig. 1) upon excitation with the $532 \mathrm{~nm}$ laser are shown in Fig. 2. Three regions are clearly discernible, corresponding to FRET (region ab), bleaching of $\mathrm{Cy} 5$ (region b-c) and bleaching of $\mathrm{Cy} 3$ (region $\mathrm{c}-\mathrm{d}$ ). Both signals exhibit fluctuations with time. In the absence of FRET, there is no correlation between donor and acceptor signals. In contrast, when FRET occurs, donor and acceptor signals exhibit a weak $(\sim 0.3)$ cross-correlation for zero lags (not shown). FRET histograms extracted from the measurements at 100 and $900 \mathrm{~ms}$ acquisition rates $\left(E_{2}\right.$, see formula 5 "Materials and methods") are shown in Fig. 3. The mean FRET efficiency (as determined form the Gaussian fit) is very similar for both acquisition rates, i.e., close to 0.58 . However, at $100 \mathrm{~ms}$ acquisition rate $(\sim 25$ photons/spot/frame on average) the distribution is wide with an apparent width (standard deviation, SD, of the Gaussian fit) of $\sim 0.205$, whereas at $900 \mathrm{~ms}$ acquisition rate ( $\sim 225$ photons/spot/frame) the distribution is narrow with apparent width (SD) of $\sim 0.10$. Due to the small photon flux, i.e., low signal-to-noise ratio and camera

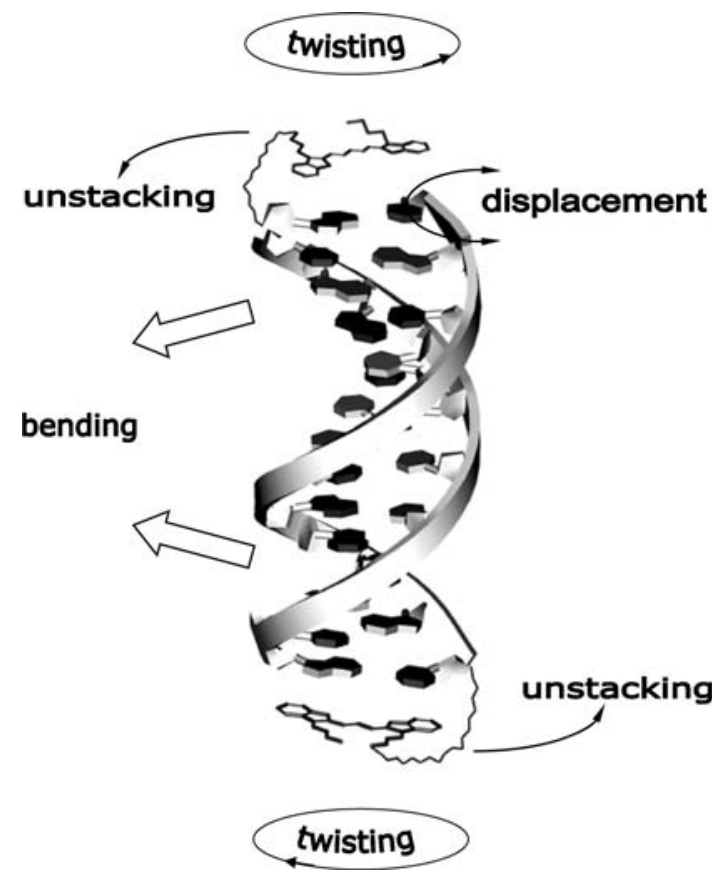

Fig. 1 Cartoon showing structure of Cy3-DNA/RNA-Cy5 molecule together with its deformations (twisting of the helix, bending of the helix, displacement of terminal base pairs, unstacking of fluorophores) accounting for broadening FRET distribution

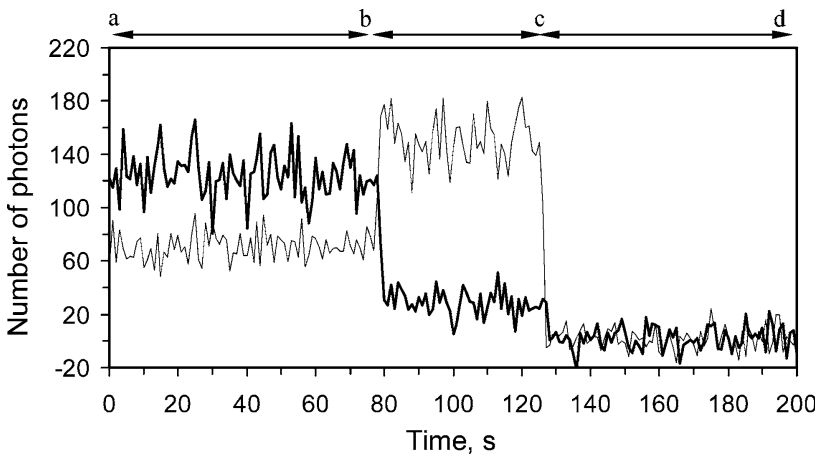

Fig. 2 Time-traces of fluorescence signals produced by the donor Cy3 (thin line) and acceptor Cy5 (thick line) linked to a 15-mer DNA/ RNA duplex upon excitation at $532 \mathrm{~nm}$ (acquisition time is $900 \mathrm{~ms}$ ). Region $a, b$ corresponds to FRET; at point $b$ the acceptor bleached; at point $c$ the donor bleached

noise, the apparent FRET efficiency can exceed the theoretical limit of one. The $\gamma$ histograms for both acquisition rates are given in Fig. 3a (inset) and 3b (inset), showing broad distributions ranging from $\sim 0.5$ to $\sim 5$ and centred around 1-1.5.

In order to understand the nature of width of FRET distribution, we analyzed the following factors which potentially contribute to the phenomenon, namely, (1) variation of the geometry of the DNA/RNA helix leading 

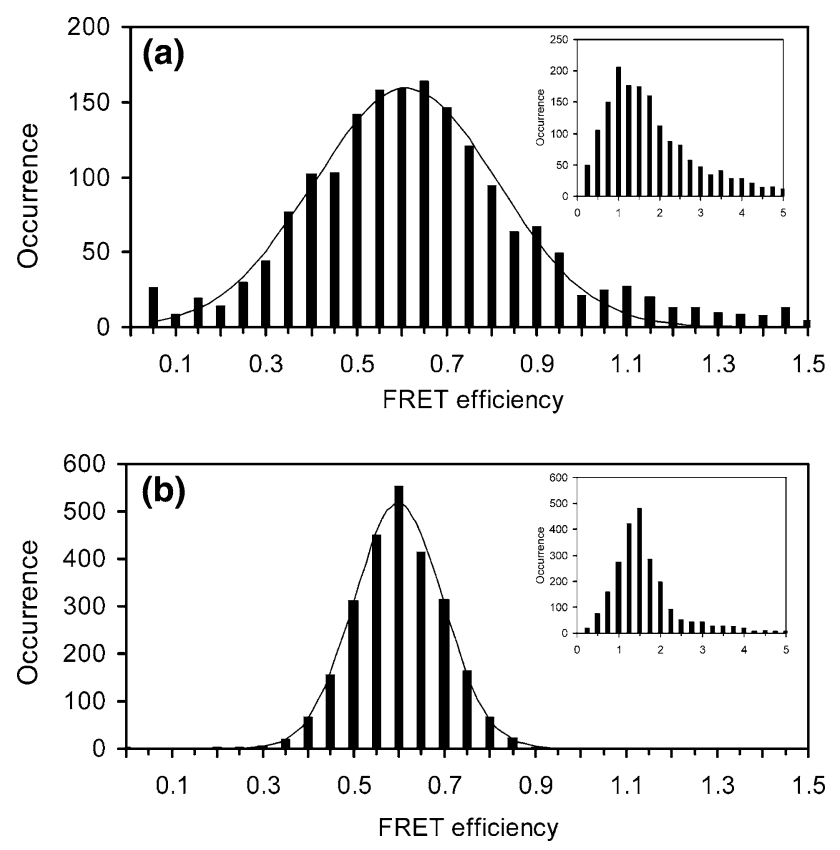

Fig. 3 FRET efficiency histograms extracted from the measurements at $100 \mathrm{~ms}$ (a) and $900 \mathrm{~ms}$ (b) acquisition times. FRET efficiency was calculated using formula 5. Each histogram was obtained from more than 2,500 measurements. Thin lines represent Gaussian fits with following parameters: $\langle E\rangle=0.58, \sigma_{E}=0.205$ (a) and $\langle E\rangle=0.58$, $\sigma_{E}=0.10$ (b). Insets represent distributions for $\gamma$ factor for either experiment calculated using formula 4

to modulations of inter-dye distance, (2) photon shot noise, (3) variation of the relative orientation of fluorophores due to helix flexibility and (4) variation of both fluorophore orientation and separation distance (Fig. 1). If not otherwise stated, we assume that both dyes stack to the end base pairs of the DNA/RNA helix, with their planes perpendicular to the helix axis (Iqbal et al. 2008a, b; Norman et al. 2000).

Contribution of DNA/RNA flexibilities to the FRET width distribution

The helix flexibilities that might contribute to the inter-dye distance could be resolved into bending flexibility, torsion rigidity and terminal base opening (base breathing). To estimate the variation of inter-dye distance due to helix bending flexibility, we assume that the DNA/RNA duplex behaves like B-form DNA with a persistence length, $P_{3 \mathrm{D}}$, of $\sim 150 \mathrm{bp}$ (Hagerman 1988). The dispersion of $<R^{2}>$ can be estimated as

$\sigma_{R^{2}, 3 \mathrm{D}}^{2}=\left\langle R_{3 \mathrm{D}}^{4}\right\rangle-\left\langle R_{3 \mathrm{D}}^{2}\right\rangle^{2}$

where $\left\langle R_{3 \mathrm{D}}^{2}\right\rangle$ and $\left\langle R_{3 \mathrm{D}}^{4}\right\rangle$ are the second and forth moments for the end-to-end distance distribution for a flexible polymer diffusing in 3D (see supplementary information).
Therefore, after expansion and leaving the major members we have

$\sigma_{R^{2}, 3 \mathrm{D}} \approx \sqrt{\frac{4}{90}} \frac{L}{P_{3 \mathrm{D}}} L^{2}$

For $L=15$ bp $\left(0.1\right.$ of $\left.P_{3 \mathrm{D}}\right) \sigma_{R^{2}, 3 \mathrm{D}}$ is $\sim 0.02 \times L^{2}$. The dispersion of $\left\langle R^{2}\right\rangle$ can be translated into dispersion of $E$ (using error propagation) leading to the following expression.

$\frac{\sigma_{E}}{E}=3(1-E) \frac{\sigma_{\left\langle R^{2}\right\rangle}}{\left\langle R^{2}\right\rangle}$

It means that for $E=0.58 \sigma_{E}$ is $\sim 0.015$.

The above considerations assume that the DNA/RNA duplex is diffusing in 3D space. However, there is a possibility that these molecules can interact with the charged surface of streptavidin (to which they are anchored via the biotinylated linker) leading to a two-dimensional (2D) configuration. Using the known expressions for $<R^{2}>_{2 \mathrm{D}}$ and $<R^{4}>_{2 \mathrm{D}}$ (see supplementary information) we have

$\sigma_{R^{2}, 2 \mathrm{D}}, \approx \sqrt{\frac{4}{45}} \frac{L}{P_{2 \mathrm{D}}} L^{2}$

In general, $P_{2 \mathrm{D}}$ is twice $P_{3 \mathrm{D}}$, therefore $\sigma_{R^{2}, 2 \mathrm{D}}$ is smaller relative to $\sigma_{R^{2}, 3 \mathrm{D}}$. However, adherence to a charged surface often results in a decrease in apparent persistence length (Podesta et al. 2005), implying that $\sigma_{R^{2}}$ can be slightly larger $(<0.02$ for $E=0.58)$.

The modulation of inter-dye distance due to torsional flexibility of the helix can be calculated using the SD for the twist angle, $\sigma_{\text {twist, }}$ of dsDNA, which is about $4 \%$ bp on average (Crothers et al. 1992). This means that the total angular displacement for the end base pairs is $\sigma_{\text {tot }}=$ $\sqrt{(N-1)} \sigma_{\text {twist }}$, i.e. $\sim 15^{\circ}$ (or $\sim 0.26$ radians) for a 15 -mer duplex. The modulation of the rise associated with helix twisting is negligible (Crothers et al. 1992), implying that the net modulation of inter-dye distance is hardly detectable for the dyes positioned close to the centre of helix axis.

For dyes positioned off the helix axis the inter-dye distance can be estimated using the following formula

$r=\sqrt{\left(L_{0}+H_{\text {rise }}(N-1)\right)^{2}+\left(L_{\mathrm{D}}^{2}+L_{\mathrm{A}}^{2}-2 L_{\mathrm{D}} L_{\mathrm{A}} \cos \varphi\right)}$

(Clegg et al. 1993),

where $r$ is the inter-dye distance, $N$ is the number of base pairs, $H_{\text {rise }}$ is the rise of DNA/RNA helix ( $H_{\text {rise }} \approx 3.0$ or $2.7 \AA$ (Han et al. 2003)) and $\varphi$ is the polar angle between donor and acceptor orientations. $L_{0}$ should be considered as the sum of the $\mathrm{Cy} 3$ and $\mathrm{Cy} 5$ rises ( $8 \AA$; Iqbal et al. 2008a) $L_{\mathrm{D}}$ and $L_{\mathrm{A}}$ can be considered as off-centre distances for donor and acceptor, respectively. Variation for $r$ due to helix rotation leads to $\sigma_{r}=\frac{L_{\mathrm{D}} L_{\mathrm{A}}}{r}|\sin \varphi| \sigma_{\text {tot }}$. Assuming that 
$L_{\mathrm{D}}$ and $L_{\mathrm{A}}$ are less than $10 \AA$ each, whereas $r$ is $\sim 46$ or $50 \AA(14 \times 2.7 \AA+8 \AA$ or $14 \times 3.0 \AA+8 \AA)$ we conclude that $\sigma_{r}$ is $<0.5 \AA$. Translating $\sigma_{r}$ into $\sigma_{E}$ using the formula

$\sigma_{E}=6 E(1-E) \frac{\sigma_{r}}{\langle r\rangle}$

gives $\sigma_{E} \sim 0.014-0.017$.

The opening of terminal base pairs or DNA breathing is characterized by the life-time $(\sim 20 \mathrm{~ms})$ and apparent dissociation constant of the non-paired bases (Nonin et al. 1995). The dissociation constant is close to unity for AT terminal base pairs (our case) and $\sim 0.015$ for GC terminal base pair. The opening will lead to the displacement of end pairs and hence modulation of inter-dye distance either (1) due to concurrent displacement of the fluorophore which is stuck to the displaced base or (2) due to unstacking of the fluorophore. Lateral displacement of the terminal base (and stacked fluorophore) can be estimated as $<5-7 \AA$. Using the above formula 12 and varying $L_{\mathrm{D}}$ with $\sigma=5 \AA$ leads to $\sigma_{r} \sim 0.7 \AA$, or $\sigma_{E} \sim 0.019$. When both dyes flip out with $\sigma=5 \AA$ each, $\sigma_{r}$ is $<1 \AA$ corresponding to $\sigma_{E} \sim 0.027$. The second case is considered in the last section.

Photon shot noise

Shot noise accounts for significant fluctuations of fluorescence signals at the single molecule level. To estimate its contribution to the experimentally extracted FRET width distribution, we modelled it following established procedures (Antonik et al. 2006; Gopich and Szabo 2005; Nir et al. 2006). When the total number of photons detected in both donor and acceptor channels is small, i.e., corresponding to the $100 \mathrm{~ms}$ acquisition rate, the FRET histogram is broad (Fig. 4a). The width (SD) of the corresponding Gaussian fit is $\sim 0.18$, close to that found experimentally $(\sim 0.205)$, implying that photon shot noise is the dominant factor accounting for the FRET width distribution. Like the experimental data, the simulated FRET distribution goes beyond the theoretical limit of one due to a low signal-to-noise ratio and camera noise. For a larger number of photons (corresponding to the $900 \mathrm{~ms}$ acquisition rate), the FRET histogram is much narrower (Fig. 4b). The width (SD) of the corresponding Gaussian fit is $\sim 0.065$, which is significantly smaller that the width of the experimental distribution $(\sim 0.10$, Fig. $3 b)$.

The absolute intensities of the donor and acceptor usually vary by $\sim 25 \%$ across the illumination area $(\sim 65 \times$ $32 \mu \mathrm{M}^{2}$ ). For these reasons in our simulations we varied the total number of photons detected in both channels accordingly. This led to a very small shift of the mean of the FRET $(<0.05)$. However, the width of the corresponding Gaussian fit varied notably either in the range
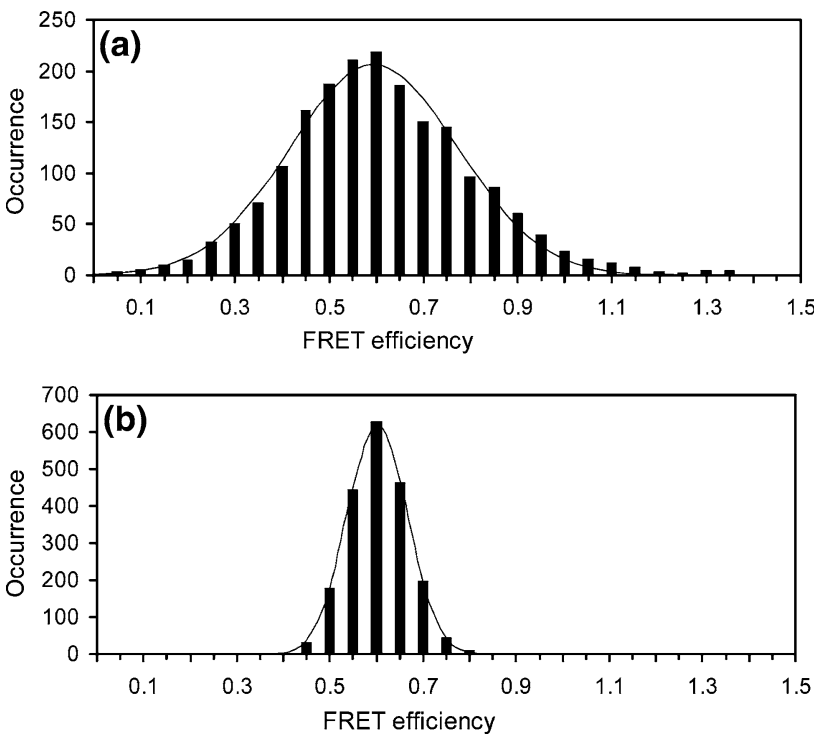

Fig. 4 FRET efficiency histograms accounting for photon shot noise and camera noise. Fluctuations of FRET efficiency were calculated as described in "Materials and methods" for 25 photons (a) and 225 photons (b) corresponding to the experimental conditions of 100 and $900 \mathrm{~ms}$ acquisition times. Thin lines represent Gaussian fits with following parameters: $\langle E\rangle=0.58, \sigma_{E} \approx 0.190$ (a) and $\langle E\rangle=$ $0.58, \sigma_{E} \approx 0.065(\mathbf{b})$

$0.170-0.195$ (corresponding to the $100 \mathrm{~ms}$ acquisition rate) or $0.055-0.075$ (corresponding to the $900 \mathrm{~ms}$ acquisition rate). This means that the increment (square root of the difference of the dispersions) of the width of the FRET distribution due to all factors other than photon shot noise is $0.065-0.11$ or $0.065-0.085$ for 100 or $900 \mathrm{~ms}$ acquisition rates, respectively.

Modulation of orientation factor due to helix bending and twisting

The above calculations assume that the orientation factor is equal to $2 / 3$, as if both fluorophores are free to move. However, bulk anisotropy measurements on the labeled duplex showed that anisotropy for $\mathrm{Cy} 3$ is $\sim 0.3$, for Cy 5 $\sim 0.25$ (direct excitation) or $\sim 0.1$ (via FRET) indicating that movement of either fluorophore is significantly hindered. Nevertheless, the relative orientation of the helix end-stacking fluorophores (and their transition moments) can be significantly modulated by helix bending and twisting.

To calculate the orientation factor for two fixed fluorophores, we followed a procedure described previously (Corry et al. 2006). Recently, it has been shown that Cy3 and Cy5 fluorophores are fixed via stacking to the terminal base pairs of DNA/RNA duplex with their transition vectors perpendicular to the helix axis (Iqbal et al. 2008a, b), forming a static angle $\varphi$ relative to each other. For 


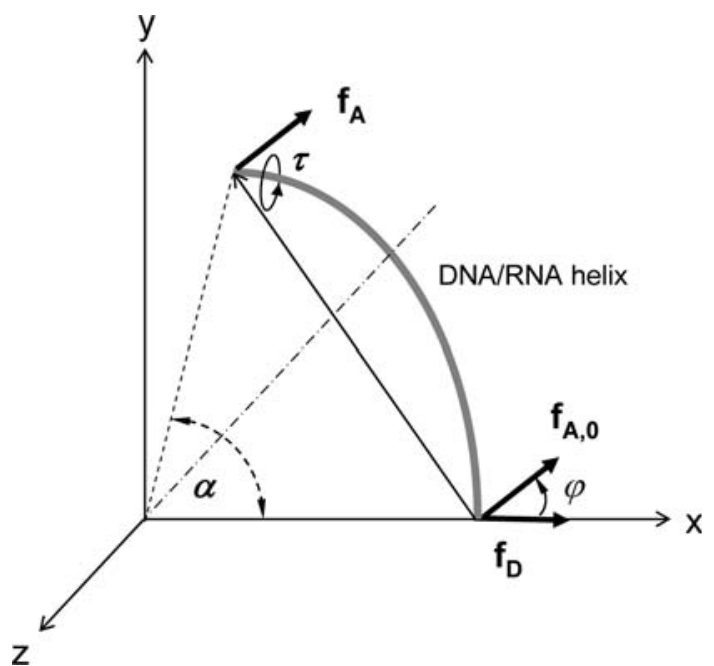

Fig. 5 Cartoon showing geometrical transformation (not to scale) of an absorption transition dipole of the acceptor upon helix bending (angle $\alpha$ ) and twisting (angle $\tau$ )

simplicity, imagine that the donor vector, is oriented along the $\mathrm{x}$ axis as shown on Fig. 5, i.e., it can be expressed as

$\mathbf{f}_{\mathbf{D}}=\left(\begin{array}{l}1 \\ 0 \\ 0\end{array}\right)$.

Initially the acceptor vector is lying in the $x z$ plane and equal to

$\mathbf{f}_{\mathbf{A}, \mathbf{0}}=\left(\begin{array}{c}\cos \varphi \\ 0 \\ -\sin \varphi\end{array}\right)$

Helix bending and twisting can be simulated by rotating the vector $\mathbf{f}_{\mathbf{A}, \mathbf{0}}$ around the axes $z$ and $y$ by angle $\alpha$ and $\tau$, corresponding to the helix bending and twisting, respectively (note that the scheme shown on Fig. 5 is not to scale as actually the angle $\alpha$ is very small). It means that the acceptor vector is equal to.

$\mathbf{f}_{\mathbf{A}}=\operatorname{rot}_{z}(\alpha) \operatorname{rot}_{y}(\tau)\left(\begin{array}{c}\cos \varphi \\ 0 \\ -\sin \varphi\end{array}\right)=\left(\begin{array}{c}\cos \alpha \cos (\varphi+\tau) \\ \sin \alpha \cos (\varphi+\tau) \\ -\sin (\varphi+\tau)\end{array}\right)$

The vector $\mathbf{r}$ is the line connecting both vectors and is defined by the helix bend as.

$\mathbf{r}=\operatorname{rot}_{z}(90+\alpha / 2)\left(\begin{array}{l}1 \\ 0 \\ 0\end{array}\right)=\left(\begin{array}{c}-\sin (\alpha / 2) \\ \cos (\alpha / 2) \\ 0\end{array}\right)$

Therefore $\quad \mathbf{f}_{\mathbf{D}} \mathbf{f}_{\mathbf{A}}=\cos \alpha \cos (\varphi+\tau), \mathbf{f}_{\mathbf{D}} r=-\sin (\alpha / 2)$ and $\quad \mathbf{f}_{\mathbf{A}} \mathbf{r}=-\sin (\alpha / 2) \cos \quad \alpha \cos (\varphi+\tau)+\cos (\alpha / 2) \sin \alpha$ $\cos (\varphi+\tau)=\sin (\alpha / 2) \cos (\varphi+\tau)$.
This means that $\kappa^{2}$ is determined by the following expression.

$\kappa^{2}=\left[\mathbf{f}_{\mathbf{D}} \mathbf{f}_{\mathbf{A}}-3\left(\mathbf{f}_{\mathbf{D}} \mathbf{r}\right)\left(\mathbf{f}_{\mathbf{A}} \mathbf{r}\right)\right]^{2}=\frac{1}{4} \cos ^{2}(\varphi+\tau)(3-\cos \alpha)^{2}$

The expression accounts for the orientation factor for values of $\alpha$ and $\tau$ at any instant. To calculate its mean and dispersion we assume that both $\alpha$ and $\tau$ are normally distributed around zero with $\mathrm{SD}$ equal to $\sigma_{\alpha}$ and $\sigma_{\tau}$, respectively (see supplementary material). $\sigma_{\alpha}$ and $\sigma_{\tau}$ relate to the $\sigma_{\text {bend }}$ and $\sigma_{\text {twist }}$ as $\sigma_{\alpha}=\sqrt{(N-1)} \sigma_{\text {bend }}$ and $\sigma_{\tau}=$ $\sqrt{(N-1)} \sigma_{\text {twist }}$, where $\sigma_{\text {bend }}$ and $\sigma_{\text {twist }}$ stand for SD for the angles between adjacent base pairs, i.e., accounting for helix bending $\left(\sim 6.6^{\circ}\right)$ and torsion $\left(4^{\circ}\right)$, respectively (Crothers et al. 1992; Schellman 1974). This permits calculation of the mean and the dispersion of the orientation factor depending on the static angle between fluorophores which are shown on Fig. 6. Note that for $\varphi=0^{\circ}$ and $\varphi=90^{\circ} \kappa^{2}$ is not equal to unity or zero due to thermal fluctuations of the helix. Analysis of twisting and bending contributions to the SD of $\kappa^{2}$ shows that the twist of the double helix has a major contribution ranging from $\sim 55$ to $\sim 90 \%$ when the $\varphi$ angle goes from $0^{\circ}$ to $90^{\circ}$ (for the given arrangements of fluorophores). The $\mathrm{SD}$ of $\kappa^{2}$ is $>0.09$ and reaches its maximum of $\sim 0.26$ for $\varphi$ angles close to $45^{\circ}$.

The FRET efficiency for any instant value of $\kappa^{2}$ can be calculated using the following expression (see supplementary information).

$E=\frac{\kappa^{2}}{\kappa^{2}+\frac{2}{3}\left(\frac{r}{R_{0, \text { free }}}\right)^{6}}$

This allows calculation of the mean of FRET and its dispersion (for any angle $\varphi$ ). Since two different structures were reported for DNA/RNA helices with rises equal to 2.7

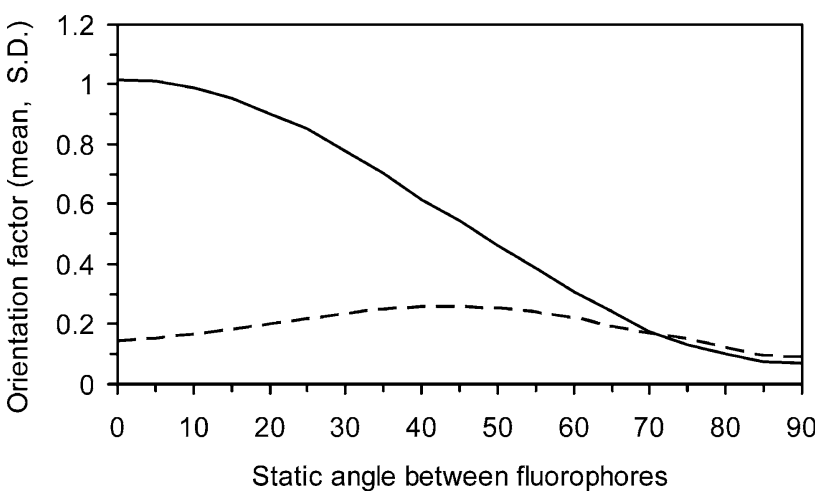

Fig. 6 Variation of orientation factor (solid line, mean) and its SD (dashed line) for the case when both fluorophores are fixed at the opposite ends of DNA/RNA helix forming a static angle $\varphi$ between transition dipoles (see supplementary information) 


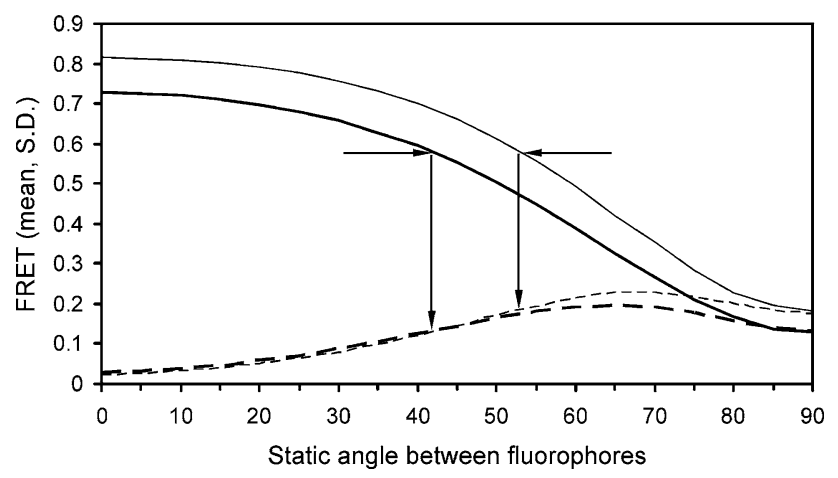

Fig. 7 FRET efficiency (solid lines) and its SD (dashed lines) calculated for the case when both fluorophores are fixed at the helix ends forming a static angle $\varphi$ between transition dipoles. FRET efficiency and SD were calculated for two DNA/RNA structures corresponding to the helix rise either $2.7 \AA$ (thin lines) or $3.0 \AA$ (thick lines), and the Förster distance was equal to $55 \AA$. Horizontal arrows point out FRET efficiency equal to 0.58 ; vertical arrows point out the corresponding values for SD and static angles for either DNA/RNA helix configuration

or $3.0 \AA$ (Han et al. 2003), we calculated the mean FRET and its SD for both cases (Fig. 7). FRET efficiency shows a monotonous decrease from $\sim 0.73-0.81$ to $0.13-0.18$ when the angle between fluorophores increases from zero to $90^{\circ}$. Likewise, $\left\langle E>\right.$ is not equal to zero for $\varphi=90^{\circ}$ due to thermal fluctuations of the helix. In contrast, the SD exhibits a bell-shaped profile with a maximum close to either $\sim 0.23$ (rise $=2.7 \AA$ ) or $\sim 0.20$ (rise $=3.0 \AA$ ). For our case $(E=0.58$, Förster distance $=55 \AA)$, the $\mathrm{SD}$ is either $\sim 0.12$ (rise $=3.0 \AA$ ) or $\sim 0.18$ (rise $=2.7 \AA$ ), implying that the static angle between the fluorophores is $\sim 42^{\circ}$ or $\sim 53^{\circ}$, respectively.

FRET efficiency for the case when one fluorophore is stacked at the helix end and the second is unconstrained

Finally, we analyzed the possibility that a fluorophore might dissociate from the terminal base pair (either spontaneously or due to breathing of terminal bases). An analysis of $\mathrm{Cy} 3$ conjugated to a DNA duplex has suggested the presence of a species with an unstacked fluorophore either in $\sim 12 \%$ (Sanborn et al. 2007) or $\sim 17 \%$ (Iqbal et al. 2008a) of molecules. With a DNA/RNA duplex the fraction of unstacked Cy3 is less ( $\sim 6 \%$ Iqbal et al. 2008a). Assuming that Cy5 behaves like the chemically similar Cy3, the total faction of the molecules with one unstacked fluorophore can be estimated as 12 (Iqbal et al. 2008a) $20 \%$. The chances that both fluorophores are unstacked are $<1 \%$ implying that the contribution of these molecules to the modulation of FRET efficiency distribution will be barely detectable. We analyzed two extreme possibilities: (1) either the orientation of the unstacked fluorophore (and its dipole moment) is coupled to the orientation of the linker or (2) the unstacked fluorophore is not restricted in its motion, which is much faster relative to the movement of the linker.

Using these simple models, we estimated the mean of energy transfer and its dispersion for both cases (see supplementary material). According to the model (1) FRET efficiency is in the range $\sim 0.36-0.40$ with $\mathrm{SD} \sim 0.36-0.38$ (linker length $=18 \AA$, helix rise $=2.7$ or $3.0 \AA$, fluorophore rise $=4 \AA$, Förster distance $=55 \AA$ ). Model (2) predicts larger values for FRET $(\sim 0.56-0.67)$ but its SD is smaller $(\sim 0.20-0.21)$. Both models predict a weak dependence for the mean $(<0.04)$ and SD $(\sim 0.02)$ on the linker length in the range 15-20 ̊. However, analysis of FRET distributions using Gaussian fitting show that these modulations would be barely discernible due to the small fraction of the molecules exhibiting alternative FRET signals. In the ultimate case, the mean of the corresponding Gaussian fit is shifted by $<2 \%$ relative to the main peak, whereas its SD may be increased $<9 \%$ (not shown).

The above calculations assume that the quantum yield of the released fluorophore remains unchanged, implying also persistence of the Förster distance $(55 \AA)$. However, recently, it was shown that the quantum yield of free $\mathrm{Cy} 3$ is $\sim 0.09$ and can be 0.39 when attached to a single-stranded DNA, or 0.16 when attached to a double-stranded DNA (Sanborn et al. 2007). These modulations of the quantum yield inevitably affect the Förster distance (Sanborn et al. 2007) and hence inter-dye separation according to the formula 1 . The corresponding calculations show noticeable modulations of the mean FRET efficiency (up to plus or minus 0.1 for either model mentioned above) with small modulations $(<0.05)$ of SD in the corresponding Gaussian fit. But still, in view of the small fraction of the molecules with one unstacked fluorophore, these modulations would be barely detected by the analysis of FRET distribution histograms.

\section{Discussion}

Single molecule experiments provide reliable tools for measuring FRET signals occurring between two fluorophores, i.e., Cy 3 and $\mathrm{Cy} 5$, attached to the opposite ends of a DNA/RNA duplex. The result of the experiment is a FRET histogram, extracted from the time series measurements, which can be analyzed using Gaussian fitting. The mean of the fit provides an estimate of the FRET efficiency, implying that this parameter has a very weak dependence on the data collection regime and intrinsic dynamics of the molecules. In contrast, the apparent width of the distribution, e.g., SD of the fit, is variable depending on the acquisition rate, dynamics of the molecules and photophysics of the FRET. 
Our data show that both the acquisition rate and dynamics of the DNA/RNA helix lead to a broadening of the FRET distribution, though to different extents. Experimental conditions (manifested mainly through the photon shot noise) are variable and their contributions can be significant (SD up to $\sim 0.19$ vs. experimental SD $\sim 0.205$ or SD up to 0.085 vs. $\sim 0.10$ ). It is clear that the contribution of the photon shot noise can be diminished by extending the acquisition time, i.e., by increasing the number of collected photons, but there are limits imposed by the fluorophores' bleaching time. The increment in the width of FRET distribution beyond the level that is governed by the photon shot noise is very similar for both acquisition times used and is in the range 0.0650.11 (100 ms acquisition rate) or $0.065-0.085$ (900 ms acquisition rate), implying that this increment is most probably determined by the structural fluctuations of the helix.

Structural fluctuations of the helix are intrinsic to it and cannot be modified by changing experimental conditions (at least on a time scale used for acquisition, which is much slower relative to any motions of a 15-mer duplex). Angular fluctuations of the dipole moments of the fluorophores account for SD close to $\sim 0.12$ (DNA/RNA helix rise $=3.0 \AA$ ) or $\sim 0.18$ (rise $=2.7 \AA$ ) as calculated for $E=0.58$ and compatible with the increment of FRET width distribution. There are other factors potentially contributing to the width of a FRET distribution. For instance, recent data show that transition of an acceptor to a triplet state may also lead to a broadening of FRET distribution, although the extent of the effect has not been quantified (Vogelsang et al. 2007). Fluctuations of interdye distance account for no more than 0.03. Taken together, we infer that angular fluctuations of the dipole moments of the fluorophores play a dominant role in determining the width of the FRET distribution, whereas fluctuations of inter-dye distance play a minor role. Hence, a simple translation of FRET width distribution into variation of inter-dye distance is likely to be erroneous.

Note that relative contribution of inter-dye distance fluctuations and angular fluctuations of dipole moments may vary depending on the way in which the dyes are attached to the helix. For instance, unstacking of one fluorophore may result in a very broad FRET distribution characterized by its SD up to $0.36-0.38$ for the linker length of $18 \AA$ (see supplementary information). In addition, helix bending and twisting may also contribute differently to FRET distribution. Our calculations show that twisting contribution to the angular fluctuations ranges from 55 to $90 \%$ for the case when both fluorophores are stacked on the helix ends. However, it is clear that the attachment of the fluorophores to the helix surface would result in a major contribution from helix bending.
According to our calculations, the static angle between the two fluorophores should be close to $\sim 42^{\circ}$ or $\sim 53^{\circ}$ (Fig. 7) depending on the structure of DNA/RNA helix. These data are corroborated by the steady-state measurements of Cy5 anisotropy through FRET which is close to $\sim 0.10$. It is known that anisotropy is related to the transfer angle between donor emission and acceptor absorption as (assuming that the transfer time is much smaller than the time needed for reorientation of dipole moments due to helix twisting) as

$r=0.4\left(\frac{3}{2} \cos ^{2} \varphi_{\mathrm{D}-\mathrm{A}}-\frac{1}{2}\right) \quad($ Dale et al. 1979)

Simple calculations show that angle $\varphi$ should be close to $\sim 45^{\circ}$. In support of our conjecture calculations of the angle between transition dipole moments of the $\mathrm{Cy} 3$ and Cy5 fluorophores stacked to the ends of DNA/RNA duplex show that this is close to $\sim 500^{\circ}-508^{\circ}$ (sum of $\mathrm{Cy} 3$ and Cy5 rotations relative to the end base pairs is equal to $62^{\circ}$, Iqbal et al. 2008a, helical twist is equal either $31.3^{\circ}$ or $31.9^{\circ}$, Han et al. 2003) or, equivalently, to $\sim 31^{\circ}-40^{\circ}$. We note that these data corroborate the assumption about stacking of $\mathrm{Cy} 3$ and $\mathrm{Cy} 5$ fluorophores to the ends of DNA/ RNA helix which are not impaired by the nature of the linkers used.

Our calculations of the average values for energy transfer (see supplementary information) presume that the experimental data also represent averaged values and therefore can be compared with each other. In support of our conjecture, first we note that the acquisition rate was always in the millisecond range. Second, changes in the orientation dipole (if free) are usually in the subnanosecond range. The rotational diffusion coefficient of the free 15mer DNA duplex is $\sim 20 \times 10^{6} \mathrm{~s}^{-1}$ (Eimer and Pecora 1991); a DNA/RNA duplex of similar length is unlikely to be very different. The rotational diffusion coefficient of a spacer of $\sim 18 \AA$ in length used for anchoring the duplex to streptavidin should be faster but its motion may be restricted by interactions of the duplex with the protein surface. Data about the collective motion of DNA/RNA helical domains arising from structural fluctuations of the duplex (bending and twisting) are almost non-existent in the literature, especially for short duplexes. However, it can be inferred that these motions mainly cover the range from tens of nanosecond to tens of microseconds (Hogan et al. 1982; Naimushin et al. 2000; Orden and Jung 2008; Porschke 2007; Schwieters and Clore 2007; Shajani and Varani 2007), although motions in the range 20-400 $\mu \mathrm{s}$ have been reported (Kojima et al. 2001). Together, these considerations indicate that in most cases, energy transfer captures a transient "static" configuration of the duplex, whereas the slow acquisition rate allows the DNA/RNA duplex and associated fluorophores to adopt all possible 
configurations within the experimental period used for data acquisition.

Acknowledgments The authors thank Dr. B. Corry for help in calculations of the orientation factor. This work was supported by a research grant from BBRSC. D. C. was awarded a Wellcome Trust VIP grant.

Open Access This article is distributed under the terms of the Creative Commons Attribution Noncommercial License which permits any noncommercial use, distribution, and reproduction in any medium, provided the original author(s) and source are credited.

\section{References}

Agrawal A, Deo R, Wang GD, Wang MD, Nie S (2008) Nanometerscale mapping and single-molecule detection with color-coded nanoparticle probes. Proc Natl Acad Sci USA 105:3298-3303. doi:10.1073/pnas.0712351105

Antonik M, Felekyan S, Gaiduk A, Seidel CA (2006) Separating structural heterogeneities from stochastic variations in fluorescence resonance energy transfer distributions via photon distribution analysis. J Phys Chem B 110:6970-6978. doi: $10.1021 / \mathrm{jp} 057257+$

Blanchard SC, Kim HD, Gonzalez RL Jr, Puglisi JD, Chu S (2004) tRNA dynamics on the ribosome during translation. Proc Natl Acad Sci USA 101:12893-12898. doi:10.1073/pnas.0403884101

Churchman LS, Okten Z, Rock RS, Dawson JF, Spudich JA (2005) Single molecule high-resolution colocalization of Cy3 and Cy5 attached to macromolecules measures intramolecular distances through time. Proc Natl Acad Sci USA 102:1419-1423. doi: 10.1073/pnas.0409487102

Clegg RM, Murchie AI, Zechel A, Lilley DM (1993) Observing the helical geometry of double-stranded DNA in solution by fluorescence resonance energy transfer. Proc Natl Acad Sci USA 90:2994-2998. doi:10.1073/pnas.90.7.2994

Coban O, Lamb DC, Zaychikov E, Heumann H, Nienhaus GU (2006) Conformational heterogeneity in RNA polymerase observed by single-pair FRET microscopy. Biophys J 90:4605-4617. doi: 10.1529/biophysj.105.078840

Conibear PB, Bagshaw CR (2000) A comparison of optical geometries for combined flash photolysis and total internal reflection fluorescence microscopy. J Microsc 200:218-229. doi:10.1046/ j.1365-2818.2000.00774.x

Corry B, Jayatilaka D, Martinac B, Rigby P (2006) Determination of the orientational distribution and orientation factor for transfer between membrane-bound fluorophores using a confocal microscope. Biophys J 91:1032-1045. doi:10.1529/biophysj.106. 080713

Crothers DM, Drak J, Kahn JD, Levene SD (1992) DNA bending, flexibility, and helical repeat by cyclization kinetics. Methods Enzymol 212:3-29. doi:10.1016/0076-6879(92)12003-9

Dale RE, Eisinger J, Blumberg WE (1979) The orientational freedom of molecular probes. The orientation factor in intramolecular energy transfer. Biophys J 26:161-193

Deniz AA, Dahan M, Grunwell JR, Ha T, Faulhaber AE, Chemla DS, Weiss S, Schultz PG (1999) Single-pair fluorescence resonance energy transfer on freely diffusing molecules: observation of Forster distance dependence and subpopulations. Proc Natl Acad Sci USA 96:3670-3675. doi:10.1073/pnas.96.7.3670

Dietrich A, Buschmann V, Muller C, Sauer M (2002) Fluorescence resonance energy transfer (FRET) and competing processes in donor-acceptor substituted DNA strands: a comparative study of ensemble and single-molecule data. J Biotechnol 82:211-231

Eimer W, Pecora R (1991) Rotational and translational diffusion of short rodlike molecules in solutiojnL: oligonucleotides. J Chem Phys 93:2324-2329. doi:10.1063/1.459904

Friedman LJ, Chung J, Gelles J (2006) Viewing dynamic assembly of molecular complexes by multi-wavelength single-molecule fluorescence. Biophys J 91:1023-1031. doi:10.1529/biophysj. 106.084004

Gell C, Brockwell D, Smith A (2006) Handbook of single molecule fluorescence spectroscopy. Oxford University Press, Oxford

Gopich I, Szabo A (2005) Theory of photon statistics in singlemolecule Förster resonance energy transfer. J Chem Phys 122:14707. doi:10.1063/1.1812746

Ha T (2001) Single-molecule fluorescence methods for the study of nucleic acids. Curr Opin Struct Biol 11:287-292. doi:10.1016/ S0959-440X(00)00204-9

Ha T (2004) Structural dynamics and processing of nucleic acids revealed by single-molecule spectroscopy. Biochemistry 43:4055-4063. doi:10.1021/bi049973s

Ha T, Rasnik I, Cheng W, Babcock HP, Gauss GH, Lohman TM, Chu S (2002) Initiation and re-initiation of DNA unwinding by the Escherichia coli Rep helicase. Nature 419:638-641. doi: 10.1038/nature01083

Hagerman PJ (1988) Flexibility of DNA. Annu Rev Biophys Biophys Chem 17:265-286. doi:10.1146/annurev.bb.17.060188.001405

Han GW, Kopka ML, Langs D, Sawaya MR, Dickerson RE (2003) Crystal structure of an RNA.DNA hybrid reveals intermolecular intercalation: dimer formation by base-pair swapping. Proc Natl Acad Sci USA 100:9214-9219. doi:10.1073/pnas.1533326100

Hogan M, Wang J, Austin RH, Monitto CL, Hershkowitz S (1982) Molecular motion of DNA as measured by triplet anisotropy decay. Proc Natl Acad Sci USA 79:3518-3522. doi:10.1073/ pnas.79.11.3518

Iqbal A, Arslan S, Okumus B, Wilson TJ, Giraud G, Norman DG, Ha T, Lilley DM (2008a) Orientation dependence in fluorescent energy transfer between $\mathrm{Cy} 3$ and $\mathrm{Cy} 5$ terminally attached to double-stranded nucleic acids. Proc Natl Acad Sci USA 105:11176-11181. doi:10.1073/pnas.0801707105

Iqbal A, Wang L, Thompson KC, Lilley DM, Norman DG (2008b) The structure of cyanine 5 terminally attached to doublestranded DNA: implications for FRET studies. Biochemistry 47:7857-7862. doi:10.1021/bi800773f

Kapanidis AN, Weiss S (2002) Fluorescent probes and bioconjugation chemistries for single-molecule fluorescence analysis of biomolecules. J Chem Phys 117:10953-10964. doi:10.1063/1. 1521158

Kapanidis AN, Lee NK, Laurence TA, Doose S, Margeat E, Weiss S (2004) Fluorescence-aided molecule sorting: analysis of structure and interactions by alternating-laser excitation of single molecules. Proc Natl Acad Sci USA 101:8936-8941. doi: 10.1073/pnas.0401690101

Kojima C, Ulyanov NB, Kainosho M, James TL (2001) Slow motion in the CAA*TTG sequence of a DNA decamer duplex studied by NMR. Biochemistry 40:7239-7246. doi:10.1021/bi0103260

Koopmans WJ, Brehm A, Logie C, Schmidt T, van Noort J (2007) Single-pair FRET microscopy reveals mononucleosome dynamics. J Fluoresc 17:785-795. doi:10.1007/s10895-007-0218-9

Kuzmenkina EV, Heyes CD, Nienhaus GU (2006) Single-molecule FRET study of denaturant induced unfolding of RNase H. J Mol Biol 357:313-324. doi:10.1016/j.jmb.2005.12.061

Lee NK, Kapanidis AN, Wang Y, Michalet X, Mukhopadhyay J, Ebright RH, Weiss S (2005) Accurate FRET measurements within single diffusing biomolecules using alternating-laser excitation. Biophys J 88:2939-2953. doi:10.1529/biophysj.104. 054114 
Merchant KA, Best RB, Louis JM, Gopich IV, Eaton WA (2007) Characterizing the unfolded states of proteins using singlemolecule FRET spectroscopy and molecular simulations. Proc Natl Acad Sci USA 104:1528-1533. doi:10.1073/pnas. 0607097104

Murphy MC, Rasnik I, Cheng W, Lohman TM, Ha T (2004) Probing single-stranded DNA conformational flexibility using fluorescence spectroscopy. Biophys J 86:2530-2537

Myong S, Stevens BC, Ha T (2006) Bridging conformational dynamics and function using single-molecule spectroscopy. Structure 14:633-643. doi:10.1016/j.str.2006.02.005

Naimushin AN, Fujimoto BS, Schurr JM (2000) Dynamic bending rigidity of a 200-bp DNA in $4 \mathrm{mM}$ ionic strength: a transient polarization grating study. Biophys J 78:1498-1518

Nir E, Michalet X, Hamadani KM, Laurence TA, Neuhauser D, Kovchegov Y, Weiss S (2006) Shot-noise limited singlemolecule FRET histograms: comparison between theory and experiments. J Phys Chem B 110:22103-22124. doi:10.1021/ jp063483n

Nonin S, Leroy JL, Gueron M (1995) Terminal base pairs of oligodeoxynucleotides: imino proton exchange and fraying. Biochemistry 34:10652-10659. doi:10.1021/bi00033a041

Norman DG, Grainger RJ, Uhrin D, Lilley DM (2000) Location of cyanine-3 on double-stranded DNA: importance for fluorescence resonance energy transfer studies. Biochemistry 39:6317-6324. doi:10.1021/bi992944a

Orden AV, Jung J (2008) Review fluorescence correlation spectroscopy for probing the kinetics and mechanisms of DNA hairpin formation. Biopolymers 89:1-16. doi:10.1002/bip.20826

Podesta A, Indrieri M, Brogioli D, Manning GS, Milani P, Guerra R, Finzi L, Dunlap D (2005) Positively charged surfaces increase the flexibility of DNA. Biophys J 89:2558-2563. doi:10.1529/ biophysj.105.064667

Porschke D (2007) Unique physical signature of DNA curvature and its implications for structure and dynamics. J Phys Chem B 111:12004-12011. doi:10.1021/jp073965e

Rueda D, Bokinsky G, Rhodes MM, Rust MJ, Zhuang X, Walter NG (2004) Single-molecule enzymology of RNA: essential functional groups impact catalysis from a distance. Proc Natl Acad Sci USA 101:10066-10071. doi:10.1073/pnas.0403575101

Sabanayagam CR, Eid JS, Meller A (2005) Using fluorescence resonance energy transfer to measure distances along individual DNA molecules: corrections due to nonideal transfer. J Chem Phys 122:061103. doi:10.1063/1.1854120

Sanborn ME, Connolly BK, Gurunathan K, Levitus M (2007) Fluorescence properties and photophysics of the sulfoindocyanine
Cy3 linked covalently to DNA. J Phys Chem B 111:11064-11074. doi:10.1021/jp072912u

Schellman JA (1974) Flexibility of DNA. Biopolymers 13:217-226. doi:10.1002/bip.1974.360130115

Schuler B, Lipman EA, Steinbach PJ, Kumke M, Eaton WA (2005) Polyproline and the "spectroscopic ruler" revisited with singlemolecule fluorescence. Proc Natl Acad Sci USA 102:2754-2759. doi:10.1073/pnas.0408164102

Schwieters CD, Clore GM (2007) A physical picture of atomic motions within the Dickerson DNA dodecamer in solution derived from joint ensemble refinement against NMR and largeangle X-ray scattering data. Biochemistry 46:1152-1166. doi: $10.1021 / \mathrm{bi061943x}$

Shajani Z, Varani G (2007) NMR studies of dynamics in RNA and DNA by $13 \mathrm{C}$ relaxation. Biopolymers 86:348-359. doi: 10.1002/bip.20650

Sugawa M, Arai Y, Iwane AH, Ishii Y, Yanagida T (2007) Single molecule FRET for the study on structural dynamics of biomolecules. Biosystems 88:243-250. doi:10.1016/j.biosystems.2006. 09.041

Thompson RE, Larson DR, Webb WW (2002) Precise nanometer localization analysis for individual fluorescent probes. Biophys $\mathbf{J}$ 82:2775-2783

Ulbrich MH, Isacoff EY (2007) Subunit counting in membrane-bound proteins. Nat Methods 4:319-321

Vogelsang J, Doose S, Sauer M, Tinnefeld P (2007) Single-molecule fluorescence resonance energy transfer in nanopipets: improving distance resolution and concentration range. Anal Chem 79:7367-7375. doi:10.1021/ac071176n

Yasuda R, Masaike T, Adachi K, Noji H, Itoh H, Kinosita K Jr (2003) The ATP-waiting conformation of rotating F1-ATPase revealed by single-pair fluorescence resonance energy transfer. Proc Natl Acad Sci USA 100:9314-9318. doi:10.1073/pnas.1637860100

Yildiz A, Selvin PR (2005) Fluorescence imaging with one nanometer accuracy: application to molecular motors. Acc Chem Res 38:574-582. doi:10.1021/ar040136s

Yim PB, Zhang X, DeJong ES, Carroll JM, Marino JP, Goldner LS (2005) Single RNA kissing complexes studied by fluorescence resonance energy transfer. In: Nicolau DV, Enderlein J, Leif RC, Farkas DL, Raghavachari R (eds) Imaging, manipulation, and analysis of biomolecules and cells: fundamentals and applications III. Proceedings SPIE 5699, pp 450-460

Zhang H, Shu D, Huang F, Guo P (2007) Instrumentation and metrology for single RNA counting in biological complexes or nanoparticles by a single-molecule dual-view system. RNA 13:1793-1802. doi:10.1261/rna.587607 